\title{
Monitoring Oxidative Degradation of Thermally Stressed Palm Olein Shortening by Spectroscopic and Chromatographic Techniques
}

\author{
Adnan Mujahid, Saba Nazir, Muhammad Hamid Raza, Azeem Intisar*, \\ Tajamal Hussain, Ahsan Sharif, Ejaz Ahmed \\ Institute of Chemistry, University of the Punjab, Quaid-i-Azam Campus, Lahore, Pakistan \\ Email: *azeemchemist@yahoo.com
}

How to cite this paper: Mujahid, A., Nazir, S., Raza, M.H., Intisar, A., Hussain, T., Sharif, A. and Ahmed, E. (2018) Monitoring Oxidative Degradation of Thermally Stressed Palm Olein Shortening by Spectroscopic and Chromatographic Techniques. Open Journal of Applied Sciences, 8, 598-606.

https://doi.org/10.4236/ojapps.2018.812048

Received: November 15, 2018

Accepted: December 21, 2018

Published: December 24, 2018

Copyright $\odot 2018$ by authors and Scientific Research Publishing Inc. This work is licensed under the Creative Commons Attribution International License (CC BY 4.0).

http://creativecommons.org/licenses/by/4.0/

\section{c) (i) Open Access}

\begin{abstract}
Deep frying with repeated use of same edible oil leads to the formation of lipid oxidation products. The presence of these oxidized products formed during frying at elevated temperature is hazardous for human health and therefore, requires prompt analysis for ensuring its fitness/quality. In this study, oxidative degradation of thermally stressed edible oils canola, sunflower and palm olein shortening was studied at $180^{\circ} \mathrm{C} \pm 5^{\circ} \mathrm{C}$ up to 48 hours. Palm olein shortening remained stable during this period as the increase in its acid value was significantly less than canola and sunflower seed oils. The shortening samples were further studied by attenuated total reflectance Fourier transformation infrared (ATR-FTIR) and gas chromatography mass spectrometry (GC-MS) to evaluate the formation of various oxidation products due to thermal stress. ATR-FTIR indicates the formation of free fatty acids (FFAs), aldehyde groups and change in degree of unsaturation while GC-MS gives important information about profile of FFAs. The combination of these analytical techniques is useful for rapid and highly sensitive analysis of oxidized edible oil thus, ensuring its quality and fitness for safe use.
\end{abstract}

\section{Keywords}

Palm Olein, Shortening, Oxidation, ATR-FTIR, GC-MS

\section{Introduction}

Vegetable seed oils are important part of daily human diet [1] [2] as they not only give characteristics texture and flavor to food but they also contain essential 
fatty acids (EFAs) [3] which possesses high calorific value and are rich in energy for examples, sunflower, soybean, canola and others. These oils are polyunsaturated fatty acids which are susceptible to oxidation [4] [5] [6] and therefore, start to decompose the moment after they are collected from seeds and this process is accelerated by environmental factors including atmospheric oxidation, humidity and temperature. Apart from this, intense and prolonged frying of different foods including vegetables and meat at elevated temperature cause thermal oxidative degradation in polyunsaturated oils. As a result of thermal stress, the physical and chemical profile of edible oils is changed drastically. Physical parameters include unpleasant odour, change in color and viscosity while chemical reactions triggered by thermal oxidation yield a variety of different products. When oxygen molecules interact with polyunsaturated oils at high temperatures, it is mainly absorbed at double bond linkages and starts the degradation process. This results in the formation of various breakdown products including hydroperoxides and a range of carbonyl compounds e.g. aldehydes, ketones, free fatty acids (FFAs) and other compounds [7]. In addition to this, the extended frying also results cis to trans isomerization. The oxidation products largely depend on oil source, its composition, thermal stress and other environmental factors.

Many of these oxidative breakdown products are dangerous and therefore, make the edible oil unhealthy for human use. Commercial fryers used edible oils for prolonged times and in this perspective it is important to determine what the oxidation products are and when to change the frying oil. In order to develop oxidation resistance edible grade, shortenings [8] [9] [10] are developed which are partially hydrogenated oils and often contain edible emulsifiers. Shortenings are resistance towards oxidation at high frying temperature and their thermal stability is an important characteristic which determines the quality of food product.

The conventional chemical tests for determining edible oil quality include acid value, peroxide value, $p$-anisidine value, iodine value and others. These tests give empirical information about chemical profile of oxidized edible oil rather giving complete data. The conventional methods are laborious as they require extensive sample preparation protocols and also consume hazardous solvents and chemicals for testing edible oil quality. In order to ensure the fitness and quality of frying oil, it is imperative to accurately and rapidly analyze [11] [12] the breakdown products in oxidized oil samples. For instance, modern spectroscopic tools such as attenuated total reflectance Fourier transformation infrared (ATR-FTIR) [13] [14] [15] [16] is another method of choice for fast and sensitive analysis of oxidized edible oil without going through lengthy sample preparation steps. The unsaturation percentage, formation of carbonyl compounds, increase in trans isomers, presence of FFAs and information about other oxidation products can be obtained from FTIR data. On the other hand, gas chromatography mass spectroscopy (GC-MS) [17] [18] is highly sensitive and sophisticated analytical method for separation and analysis of volatile compounds produces during thermal oxidation process. 
In this work, we analyzed canola, sunflower and palm olein shortening by conventional volumetric method for monitoring acid values of these samples due to thermal stress. In the next phase, palm olein shortening was analyzed by ATR-FTIR to observe the change in chemical profile of oil including degree of unsaturation, formation of FFAs and aldehyde compounds. GC-MS investigation revealed the nature and composition of FFAs produced during thermal oxidation. The use of ATR-FTIR and GC-MS is suitable for analyzing breakdown products in thermally stressed palm olein shortening and thus, accessing its quality in quick time without extensive sample preparation steps.

\section{Experimental}

\subsection{Reagents and Chemicals}

All the chemicals and reagents used for determination of acid value were obtained from Riedel-de-Haën and Merck and used as received. These chemicals include potassium hydroxide $(\mathrm{KOH})$, phenolphthalein $\left(\mathrm{C}_{20} \mathrm{H}_{14} \mathrm{O}_{4}\right)$, isopropyl alcohol $\left(\mathrm{C}_{3} \mathrm{H}_{8} \mathrm{O}\right)$ and oxalic acid $\left(\mathrm{C}_{2} \mathrm{H}_{2} \mathrm{O}_{4}\right)$.

\subsection{Thermal Treatment of Edible Oil Samples}

The oil samples were heated from 0 - 48 hours at $180^{\circ} \mathrm{C} \pm 5^{\circ} \mathrm{C}$ on hotplate under continuous magnetic stirring to ensure uniform heating. The heated oil samples were collected after different time intervals and stored in air tight glass tubes to avoid contamination.

\subsection{Acid Value Determination}

The acid values of thermally stressed sunflower, canola and palm olein shortening were determined by volumetric method. For the determination of acid value, $5 \mathrm{gm}$ of oil sample was dissolved in $50 \mathrm{~mL}$ of isopropyl alcohol in a conical flask and then 2 - 3 drops of phenolphthalein as indicator was added. The contents of flask were titrated against standardized $0.05 \mathrm{~N} \mathrm{KOH}$ till the pink color persists for 10 seconds which is considered as end point. The formula used for calculating acid value is described as follows.

$$
\text { Acid Value }=\frac{56.1 \times V \times N}{W}
$$

In this expression, $V$ represents the volume $(\mathrm{mL})$ of $\mathrm{KOH}, N$ is the normality of $\mathrm{KOH}$ and $W$ indicates the weight (gm) of oil sample.

\subsection{ATR-FTIR Studies}

Thermally stressed palm olein shortening samples were analyzed by Nicolet 6700 ATR-FTIR spectrometer. All the spectra were recorded in absorbance mode in the range from 650 to $4000 \mathrm{~cm}^{-1}$.

\subsection{GC-MS Analysis}

The chemical composition of untreated and thermally oxidized palm olein 
shortening samples was analyzed by JOEL GC-MS model JMS-A $\times 5050 \mathrm{H}$ mass spectrometer (JOEL, Japan) coupled with Hewlett Packard 5890 gas chromatograph. Following conditions were maintained for GC-MS analysis of shortening samples i.e. helium as carrier gas, split ratio 1:100, electrical energy $70 \mathrm{eV}$, ionization current $200 \mu \mathrm{A}$, ionization source temperature $250^{\circ} \mathrm{C}$, column temperature with $6^{\circ} \mathrm{C} / \mathrm{min}$ rise to $230^{\circ} \mathrm{C}$. The comparative GC-MS data of untreated and thermally treated shortening samples for 48 hours was used to determine the formation of breakdown products due to oxidation. The chemical constituents of oxidized palm olein shortening were identified by their retention time and compared with NIST147.LIB.

\section{Results and Discussion}

The acid values determined for canola, sunflower and palm olein shortening are displayed in Figure 1. In this graph it can be seen that acid values for canola and sunflower oil increases considerably as the heating time increases indicating that thermal stress results significant oxidation of edible oils. While palm olein shortening remains stable for quite long time and the increase in acid value is much less as compare to canola and sunflower oil samples. This clearly demonstrates that palm olein shortening has good resistance against thermal oxidation.

In the next phase, two palm olein shortening samples heated for 24 hours and 48 hours at $180^{\circ} \mathrm{C} \pm 5^{\circ} \mathrm{C}$ were analyzed by ATR-FTIR and their spectra were compared with untreated shortening sample, the recorded spectra are shown in Figure 2. The fingerprint region of FTIR i.e. $1650-650 \mathrm{~cm}^{-1}$ is more complex as it contains more peaks than functional groups region i.e. $4000-1650 \mathrm{~cm}^{-1}$. In this spectrum of untreated shortening, the absorbance at $721 \mathrm{~cm}^{-1}$ is related to rocking vibrations of $-\left(\mathrm{CH}_{2}\right)_{\mathrm{n}}$ - functional groups of long chain fatty acids whereas a small weak absorbance peak at $868 \mathrm{~cm}^{-1}$ is related to $=\mathrm{CH}_{2}$ wagging vibrations.

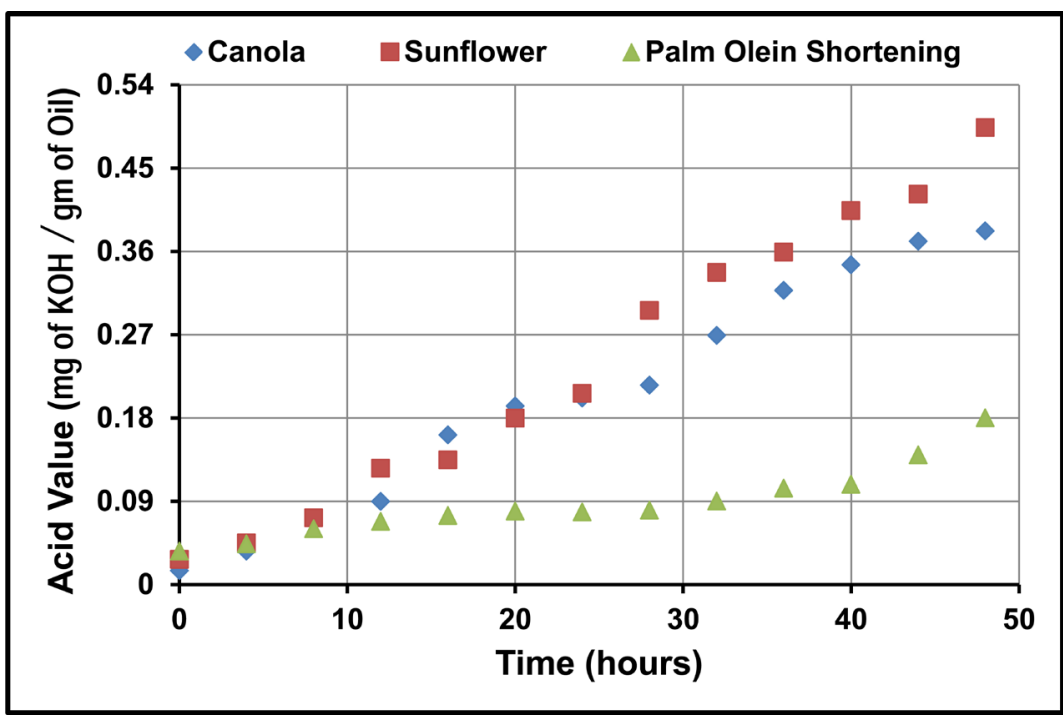

Figure 1. Comparison of acid values of canola, sunflower and palm olein shortening as function of heating time, all the samples were heated at $180^{\circ} \mathrm{C} \pm 5^{\circ} \mathrm{C}$. 


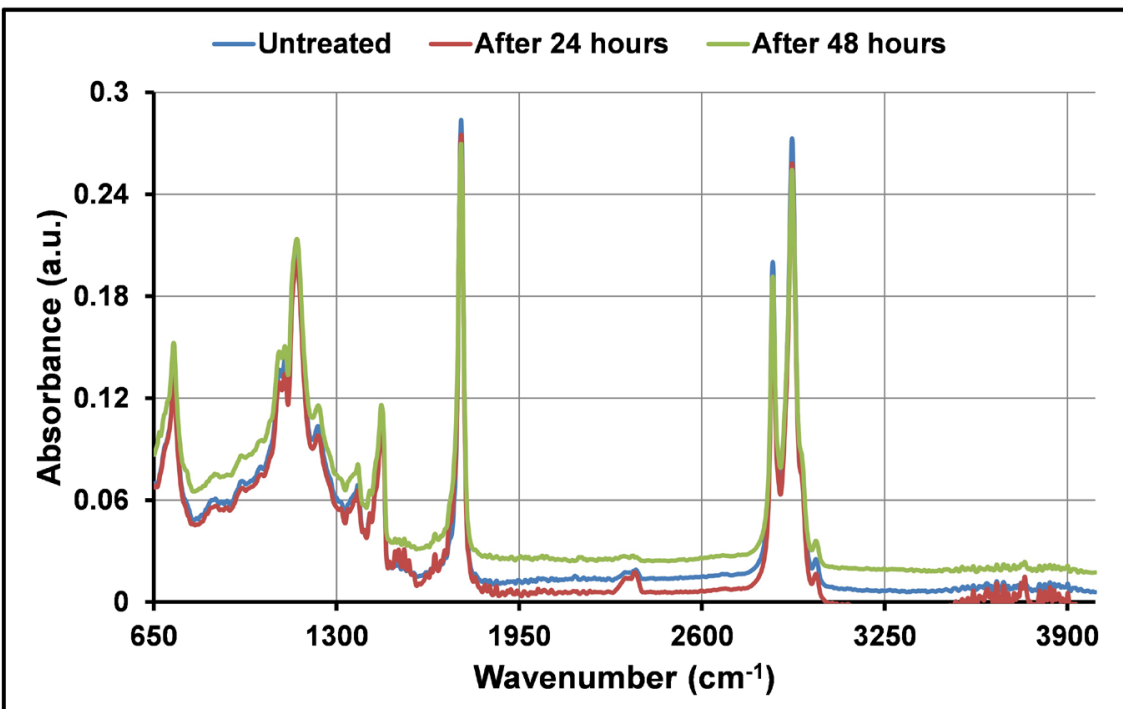

Figure 2. FTIR spectra of untreated, 24 and 48 hours heated shortening samples at $180^{\circ} \mathrm{C}$ $\pm 5^{\circ} \mathrm{C}$.

Out of plane bending vibrations of $-\mathrm{CH}=\mathrm{CH}-($ cis $)$ and $-\mathrm{CH}=\mathrm{CH}-$ (trans) groups can be observed at $911 \mathrm{~cm}^{-1}$ and $962 \mathrm{~cm}^{-1}$, respectively. Ester groups of fatty acids showed strong absorbance band at $1158 \mathrm{~cm}^{-1}$ due to stretching vibrations of $-\mathrm{C}-\mathrm{O}$ furthermore, different modes of bending vibrations of $\mathrm{CH}_{2}$ and $\mathrm{CH}_{3}$ groups can be observed around $1375 \mathrm{~cm}^{-1}$ and $1459 \mathrm{~cm}^{-1}$. A small peak at $1650 \mathrm{~cm}^{-1}$ indicates the $\mathrm{C}=\mathrm{C}$ cis stretching vibrations while a strong and intense peak of carbonyl functional groups of triglycerides is shown at $1743 \mathrm{~cm}^{-1}$. Absorbance in the range of $2852 \mathrm{~cm}^{-1}$ and $2920 \mathrm{~cm}^{-1}$ shows $\mathrm{CH}_{2}$ symmetric and asymmetric stretching vibrations, respectively. And finally, absorbance at 3005 $\mathrm{cm}^{-1}$ was assigned to $=\mathrm{C}-\mathrm{H}$ stretching vibration of cis double bond.

The FTIR data of untreated shortening is in accordance with the reported literature values [13] [14] [19] [20] however, after heating for 24 and 48 hours at $180^{\circ} \mathrm{C} \pm 5^{\circ} \mathrm{C}$, there are some obvious changes in certain regions of spectra. For instance, from Figure 3 it can be seen that after heating the samples, the absorbance peak of carbonyl functional groups showed broadening comparing to untreated sample. The broadening in carbonyl peak is due to the formation of aldehyde groups or other secondary oxidation products resulted from thermal oxidation. In addition to peak broadening, the heated samples also showed shoulder peaks around $1700 \mathrm{~cm}^{-1}$ which corresponds to FFAs production due to oxidation. The formation of FFAs during frying is hazardous for human health and is easily identified by FTIR spectra. And furthermore, the disappearance of absorbance peak around $1650 \mathrm{~cm}^{-1}$ i.e. related to $\mathrm{C}=\mathrm{C}$ stretching vibrations indicates the decrease in degree of unsaturation due to thermal oxidation.

The thermal oxidation of palm olein shortening is also indicated by decrease in stretching vibrations of $\mathrm{C}-\mathrm{H}$ groups of $\mathrm{CH}_{2}$ as shown in Figure 4. It can be seen that absorbance peak corresponding $-\mathrm{CH}_{2}$ asymmetric stretching vibrations tends to decrease from fresh to oxidized samples. This decrease in absorbance of 


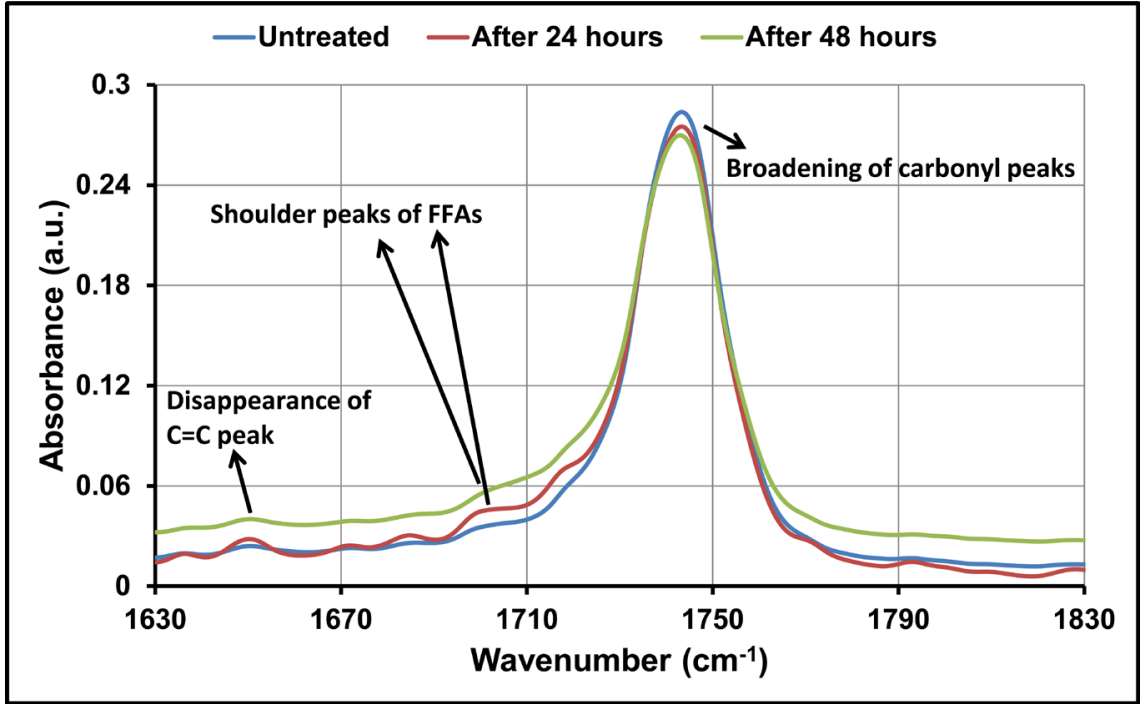

Figure 3. FTIR spectra of untreated, 24 and 48 hours heated samples at $180^{\circ} \mathrm{C} \pm 5^{\circ} \mathrm{C}$ to observe broadening in carbonyl groups peak, production of FFAs and disappearance of $\mathrm{C}=\mathrm{C}$ stretching vibrations.

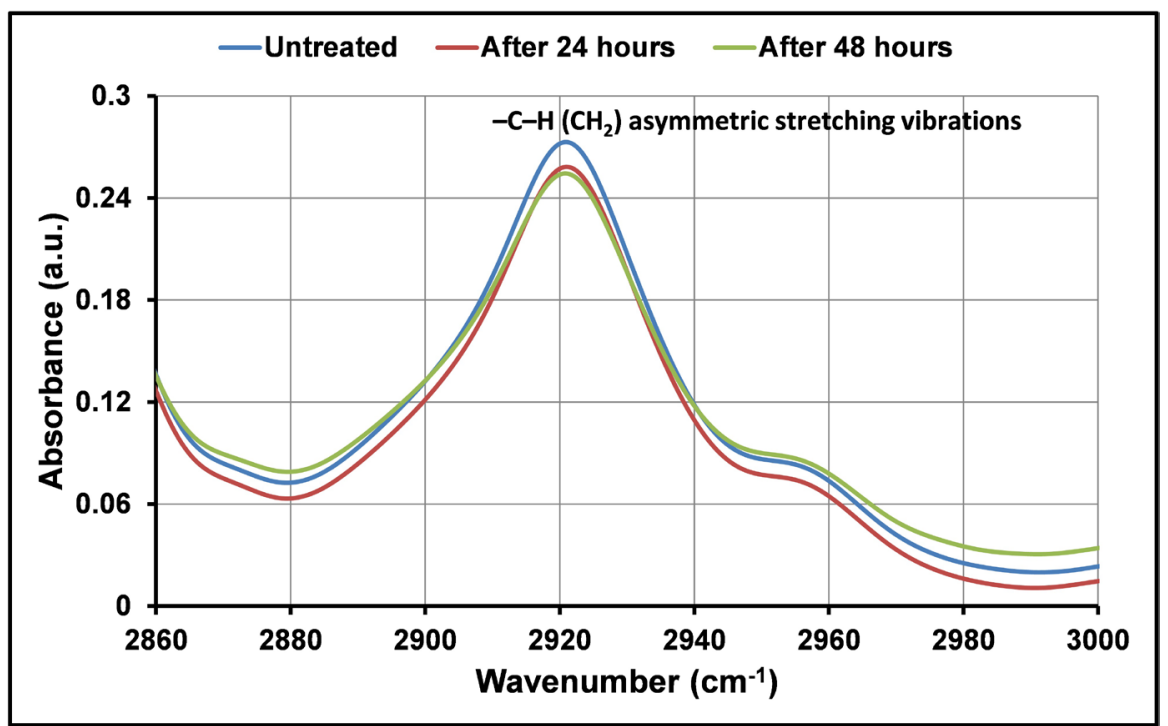

Figure 4. FTIR spectra of untreated, 24 and 48 hours heated samples at $180^{\circ} \mathrm{C} \pm 5^{\circ} \mathrm{C}$ showing decrease in absorbance intensity of $-\mathrm{C}-\mathrm{H}\left(\mathrm{CH}_{2}\right)$ asymmetric stretching vibrations.

stretching vibrations of $-\mathrm{CH}_{2}$ functional groups suggests the oxidation in palm olein shortening.

In next phase, the untreated and thermally oxidized palm olein shortening samples were analyzed by GC-MS to evaluate the nature and composition of fatty acids profile. It was observed that palm olein shortening heated at 48 hours contains mixture of saturated and unsaturated FFAs as major volatile components as the data is shown in Table 1 . These include $\mathrm{n}$-hexadecanoic acid (palmitic acid) and octadecanoic acid (stearic acid) as saturated fatty acids while 9-octadecenoic acid (oleic acid) as monounsaturated fatty acids. In case of 
Table 1. GC-MS data of 48 hours heated palm olein shortening sample to observe FFAs produced due to thermal oxidation.

\begin{tabular}{ccccc}
\hline Sr. No. & Compound Name & $\begin{array}{c}\text { Retention } \\
\text { Time (min.) }\end{array}$ & $\begin{array}{c}\text { Molecular } \\
\text { Weight }\end{array}$ & $\begin{array}{c}\text { Relative } \\
\text { Abundance (\%) }\end{array}$ \\
\hline $\mathbf{1}$ & Palmitic Acid (saturated fatty acid) & 16.521 & 256 & 22.34 \\
$\mathbf{2}$ & Oleic Acid (monounsaturated fatty acid) & 17.757 & 282 & 45.15 \\
3 & Stearic Acid (saturated fatty acid) & 17.860 & 284 & 7.07 \\
\hline
\end{tabular}

untreated sample, no FFAs were found. GC-MS provided important information about FFAs profile formed due to thermal oxidation.

\section{Conclusion}

Palm olein shortening samples are much more stable as they showed superior resistance towards oxidation comparing to canola and sunflower oils. The conventional volumetric methods for accessing the fitness and quality of edible oils are tedious, lengthy and they only offer empirical information about oxidation products. ATR-FTIR provided detailed chemical profile of degradation products as a result of thermal oxidation in relatively much faster and easier way without extensive laborious laboratory work and sample preparation steps. The formation of FFAs, degree of unsaturation and other related information of thermally stressed edible oil can be readily obtained. While GC-MS results provided important information about the profile of FFAs formed due to thermal oxidation. The combined data of ATR-FTIR and GC-MS techniques is useful for rapid and highly sensitive analysis of oxidized edible oils thus, monitoring its quality for healthy use.

\section{Acknowledgements}

Authors are thankful to Department of Chemistry, GC University Lahore for GC-MS analysis of palm olein shortening.

\section{Conflicts of Interest}

The authors declare no conflicts of interest regarding the publication of this paper.

\section{References}

[1] Caughey, G.E., Mantzioris, E., Gibson, R.A., Cleland, L.G. and James, M.J. (1996) The Effect on Human Tumor Necrosis Factor Alpha and Interleukin 1 Beta Production of Diets Enriched in N-3 Fatty Acids from Vegetable Oil or Fish Oil. The American Journal of Clinical Nutrition, 63, 116-122.

https://doi.org/10.1093/ajcn/63.1.116

[2] Dubois, V., Breton, S., Linder, M., Fanni, J. and Parmentier, M. (2007) Fatty Acid Profiles of 80 Vegetable Oils with Regard to their Nutritional Potential. European Journal of Lipid Science and Technology, 109, 710-732.

https://doi.org/10.1002/ejlt.200700040 
[3] Simopoulos, A.P. (1999) Essential Fatty Acids in Health and Chronic Disease. The American Journal of Clinical Nutrition, 70, 560s-569s. https://doi.org/10.1093/ajcn/70.3.560s

[4] Spatari, C., De Luca, M., Ioele, G. and Ragno, G. (2017) A Critical Evaluation of the Analytical Techniques in the Photodegradation Monitoring of Edible Oils. LWT-Food Science and Technology, 76, 147-155. https://doi.org/10.1016/j.lwt.2016.10.055

[5] Moreno, M.M., Olivares, D.M., Lopez, F.A., Adelantado, J.G. and Reig, F.B. (1999) Determination of Unsaturation Grade and Trans Isomers Generated during Thermal Oxidation of Edible Oils and Fats by FTIR. Journal of Molecular Structure, 482, 551-556. https://doi.org/10.1016/S0022-2860(98)00937-5

[6] Choe, E. and Min, D.B. (2006) Mechanisms and Factors for Edible Oil Oxidation. Comprehensive Reviews in Food Science and Food Safety, 5, 169-186. https://doi.org/10.1111/j.1541-4337.2006.00009.x

[7] Moreno, M.M., Olivares, D.M., López, F.A., Martínez, V.P. and Reig, F.B. (1999) Study of the Formation of Carbonyl Compounds in Edible Oils and Fats by ${ }^{1} \mathrm{H}-\mathrm{NMR}$ and FTIR. Journal of Molecular Structure, 482, 557-561. https://doi.org/10.1016/S0022-2860(98)00938-7

[8] Cheong, L.Z., Tan, C.P., Long, K., Yusoff, M.S.A., Arifin, N., Lo, S.K. and Lai, O.M. (2007) Production of a Diacylglycerol-Enriched Palm Olein using Lipase-Catalyzed Partial Hydrolysis: Optimization Using Response Surface Methodology. Food Chemistry, 105, 1614-1622. https://doi.org/10.1016/j.foodchem.2007.03.070

[9] Jin, Q., Zhang, T., Shan, L., Liu, Y. and Wang, X. (2008) Melting and Solidification Properties of Palm Kernel Oil, Tallow, and Palm Olein Blends in the Preparation of Shortening. Journal of the American Oil Chemists' Society, 85, 23-28. https://doi.org/10.1007/s11746-007-1152-3

[10] Nor Aini, I. and Miskandar, M.S. (2007) Utilization of Palm Oil and Palm Products in Shortenings and Margarines. European Journal of Lipid Science and Technology, 109, 422-432. https://doi.org/10.1002/ejlt.200600232

[11] Herrera, L.C., Ramaley, L., Potvin, M.A. and Melanson, J.E. (2013) A Method for Determining Regioisomer Abundances of Polyunsaturated Triacylglycerols in Omega-3 Enriched Fish Oils Using Reversed-Phase Liquid Chromatography and Triple-Stage Mass Spectrometry. Food Chemistry, 139, 655-662. https://doi.org/10.1016/j.foodchem.2012.12.059

[12] Xu, L., Yu, X., Liu, L. and Zhang, R. (2016) A Novel Method for Qualitative Analysis of Edible Oil Oxidation Using an Electronic Nose. Food Chemistry, 202, 229-235. https://doi.org/10.1016/j.foodchem.2016.01.144

[13] Pinto, R.C., Locquet, N., Eveleigh, L. and Rutledge, D.N. (2010) Preliminary Studies on the Mid-Infrared Analysis of Edible Oils by Direct Heating on an ATR Diamond Crystal. Food Chemistry, 120, 1170-1177. https://doi.org/10.1016/j.foodchem.2009.11.053

[14] Vlachos, N., Skopelitis, Y., Psaroudaki, M., Konstantinidou, V., Chatzilazarou, A. and Tegou, E. (2006) Applications of Fourier Transform-Infrared Spectroscopy to Edible Oils. Analytica Chimica Acta, 573, 459-465.

https://doi.org/10.1016/j.aca.2006.05.034

[15] Tay, A., Singh, R.K., Krishnan, S.S. and Gore, J.P. (2002) Authentication of Olive Oil Adulterated with Vegetable Oils Using Fourier Transform Infrared Spectroscopy. LWT-Food Science and Technology, 35, 99-103. https://doi.org/10.1006/fstl.2001.0864 
[16] Rohman, A. and Man, Y.C. (2010) Fourier Transform Infrared (FTIR) Spectroscopy for Analysis of Extra Virgin Olive Oil Adulterated With Palm Oil. Food Research International, 43, 886-892. https://doi.org/10.1016/j.foodres.2009.12.006

[17] Guo, L., Xie, M.Y., Yan, A.P., Wan, Y.Q. and Wu, Y.M. (2006) Simultaneous Determination of Five Synthetic Antioxidants in Edible Vegetable Oil by GC-MS. Analytical and Bioanalytical Chemistry, 386, 1881.

https://doi.org/10.1007/s00216-006-0738-1

[18] Beltrán, A., Ramos, M., Grané, N., Martín, M.L. and Garrigós, M.C. (2011) Monitoring the Oxidation of Almond Oils by HS-SPME-GC-MS and ATR-FTIR: Application of Volatile Compounds Determination to Cultivar Authenticity. Food Chemistry, 126, 603-609. https://doi.org/10.1016/j.foodchem.2010.11.058

[19] Christy, A.A. and Egeberg, P.K. (2006) Quantitative Determination of Saturated and Unsaturated Fatty Acids in Edible Oils by Infrared Spectroscopy and Chemometrics. Chemometrics and Intelligent Laboratory Systems, 82, 130-136.

https://doi.org/10.1016/j.chemolab.2005.06.019

[20] Zhang, Q., Liu, C., Sun, Z., Hu, X., Shen, Q. and Wu, J. (2012) Authentication of Edible Vegetable Oils Adulterated with Used Frying Oil by Fourier Transform Infrared Spectroscopy. Food Chemistry, 132, 1607-1613.

https://doi.org/10.1016/j.foodchem.2011.11.129 